\title{
Síndrome de Burnout : estudo comparativo entre professores do Ensino Especial e do Ensino Regular
}

\author{
Ana Claudia Braun \\ Pontifícia Universidade Católica do Rio Grande do Sul, RS \\ Mary Sandra Carlotto \\ Pontifícia Universidade Católica do Rio Grande do Sul, RS
}

\begin{abstract}
Resumo
O objetivo deste estudo foi verificar se existem diferenças nos índices das dimensões de SB, Perfil 1 e 2 entre professores de ensino especial e regular. A amostra constituiu-se de 160 professores, 80 de ensino especial e 80 de ensino regular. Os instrumentos utilizados foram um questionário elaborado para levantamento de dados demográficos e laborais e o Cuestionario para la Evaluacióndel Síndrome de Quemarse por elTrabajo - CESQT. Os resultados encontrados, por meio do teste t de student, evidenciaram que professores de ensino especial possuem média mais elevada na dimensão de llusão pelo Trabalho e professores de ensino regular, maiores índices médios de Perfil 1 e Perfil 2 de Burnout. Resultados apontam a necessidade de intervenções diferenciadas que considerem as peculiaridades funcionais dos grupos investigados.
\end{abstract}

Palavras-chave: Professores, stress ocupacional, ensino.

\section{Burnout Syndrome: comparative study between teacher of special education and regular education}

\begin{abstract}
In this study we propose to determine whether there is a difference in the rates of the dimensions of BS and Profile 1 and Profile 2 among special education and regular teachers. The sample consisted of 160 teachers, 80 in special education and 80 in regular education. We used a questionnaire to survey demographic and professional data and the Cuestionariopara La Evaluación del Síndrome de Quemarsepor el Trabajo CESQT to evaluate Burnout Syndrome, as instruments. The results obtained by means of the Student's $t$ test show that special education teachers have higher averages in the dimension of Enthusiasm toward the job, and regular education teachers have higher average rates of Profile 1 and Profile 2 of Burnout Syndrome. Results point to the need for different interventions that consider the peculiarities of the investigated groups.
\end{abstract}

Keywords: Teachers, occupational stress, teaching.

\section{Síndrome de Burnout: estudio comparativo entre profesores de educación} especial y regular

\section{Resumen}

El objetivo de este estudio fue verificar si existen diferencias en índices de dimensiones de SB, Perfil 1 e 2 entre profesores de educación especial y regular. La muestra fue compuesta por 160 profesores, 80 de educación especial y 80 de educación regular. Los instrumentos utilizados fueron un cuestionario elaborado para levantar datos demográficos y laborales y el Cuestionario para la Evaluación del Síndrome de Quemarse por el Trabajo - CESQT. Los resultados encontrados por medio del test $t$ de student mostraron que profesores de educación especial tienen promedio más elevado en la dimensión de llusión por el Trabajo, en cuanto que los profesores de educación regular, mayores índices promedio de Perfil 1 y Perfil 2 de Burnout. Los resultados señalan para la necesidad de intervenciones diferenciadas que considere las peculiaridades funcionales de los grupos investigados.

Palabras Clave: Profesores, estrés ocupacional, ensenanza. 


\section{Introdução}

A Síndrome de Burnout (SB) é um fenômeno psicossocial que surge como resposta a estressores crônicos no trabalho, em profissionais que lidam com clientes de forma emocional, direta e constante (Gil-Monte, 2005; Maslach, 2003; Maslach, Schaufeli, \& Leiter, 2001). No Brasil, desde 1999 a SB é reconhecida como doença profissional, pelo Decreto-Lei 6042/07, da Previdência Social, incluída na lista B do grupo V, do CID 10.

Segundo o modelo de Gil-Monte (2005), Burnout é representada por quatro dimensões, sendo elas: 1) Ilusão pelo trabalho, indicando o desejo individual de atingir metas relacionadas ao trabalho percebidas pelo sujeito como atraentes e fonte de satisfação pessoal; 2) Desgaste psíquico, caracterizado pelo sentimento de exaustão emocional e física em relação ao contato direto com pessoas que são fonte ou causadoras de problemas; 3) Indolência, evidenciada pela presença de atitudes de indiferença em relação a pessoas que necessitem ser atendidas no ambiente de trabalho, assim como insensibilidade aos problemas alheios; 4) Culpa, caracterizada pelo surgimento de cobrança e sentimento de culpabilização sobre o comportamento e atitudes negativas desenvolvidas no trabalho.

Neste modelo teórico, Gil-Monte (2005) delimitou dois perfis possíveis de Burnout. O Perfil 1 é caracterizado pela presença de sentimentos e atitudes em relação ao estresse laboral que está sendo vivenciado geradoras de mal-estar moderado. O sujeito ainda está inserido em seu ambiente de trabalho, porém poderia desenvolver melhor suas atividades laborais. Por sua vez, o Perfil 2 inclui os sentimentos citados anteriormente, acrescidos de culpa. Estes são considerados casos clínicos mais deteriorados e podem ocasionar afastamento do trabalho e a presença de comorbidades psiquiátricas.

Embora investigado em diversas categorias profissionais, cresce nos últimos anos o reconhecimento de que a SB na categoria docente é um problema de saúde pública (Batista, Carlotto, Coutinho, \& Augusto, 2010), em face da gravidade dos danos à saúde do trabalhador (Carlotto, 2010; OMS, 1994; Pocinho \& Perestelo, 2011). Segundo Yong e Yue (2007), a SB tem efeitos negativos para o professor, tais como rompimento com os hábitos normais, perda do entusiasmo e da criatividade, incapacidade para se concentrar e reações exageradas para moderar o estresse. Os efeitos da SB, em curto prazo, consistem em menor autocontrole, baixo autorrespeito, pouca eficiência no trabalho e alto nível de irritabilidade. Os efeitos, em longo prazo, são depressão, possibilidade de úlcera, hipertensão e alcoolismo. A SB, além de afetar o clima escolar e diminuir o moral dos professores, impede a realização dos objetivos educacionais e aumenta a probabilidade de abandono da profissão.

A profissão docente está exposta a uma série de estressores psicossociais que, se persistentes, podem levar à SB (Gasparini, Barreto, \& Assunção, 2006; Gomes, Montenegro, Peixoto, \& Peixoto, 2010). Segundo Leite e Souza (2007), no que se refere aos problemas relacionados às condições de trabalho, há consenso na literatura quanto aos diversos problemas enfrentados pelos professores. Os autores referem estudos nos quais são destacados aspectos como: precárias condições de trabalho (iluminação, espaço físico, material didático); organização do trabalho (multiplicidade de tarefas diferenciadas, ritmos de trabalho, aumento das exigências cognitivas, perda de autonomia, burocratização, rotinização das atividades educativas); aspectos físicos (posturas desconfortáveis, uso elevado e frequente da voz, peso de grandes quantidades de materiais); aspectos relacionais (falta de diálogo com a administração e equipe técnica, dificuldades nas relações com as famílias dos alunos, falta de valorização do trabalho realizado, estado psicológico, disciplina de alunos); e aspectos sociais (baixos salários, trabalho em mais de uma escola, imagem errônea da opinião pública sobre o professor).

Professores de diferentes níveis de ensino assemelham-se em algumas atribuições e diferenciam-se em outras. Das atribuições gerais, emergem características que os fazem ser reconhecidos como categoria profissional, porém outras Ihe são específicas em função de determinados contextos (Guglielmi \& Tatrow, 1998). De acordo com os autores, o trabalho desenvolvido apresenta diferentes desafios, demandas e recompensas, dependendo de variáveis como tipo de escola - pública ou privada, urbana ou rural -, faixa etária dos alunos e o contexto social onde está inserido. Rudow (1999) destaca a importância do estudo da relação entre Síndrome de Burnout e o tipo de escola na qual o professor atua, tanto em termos transculturais como nos aspectos relacionados ao nível de ensino. As demandas e a sobrecarga de trabalho diferem consideravelmente de uma escola para outra.

Um estudo de R. Zabel e M. Zabel (2001), desenvolvido com o objetivo de identificar diferenças nas variáveis preditoras de Burnout de acordo com o tipo de ensino, aponta algumas semelhanças e diferenças nos estressores de professores em geral e em relação aos professores de ensino especial. Como semelhança, indica a sobrecarga de trabalho, e como fatores de estresse diferenciados, a falta de apoio dos pais e da administração, que implicam de forma direta na motivação de professores de Ensino Especial. Outros preditores identificados foram falta de clareza na atribuição das funções, falta de apoio dos colegas, conflitos escolares (Crane \& Iwanicki, 1986), dificuldades de aprendizagem, falta de condições, carência de materiais de trabalho adequados aos alunos (R. Zabel \& M. Zabel, 2001) e falha administrativa na elaboração e implementação de atividades e planos de ensino (Cherniss, 1980). Outro estudo enfatizou características organizacionais como importantes fatores que contribuem para a SB em docentes, tais como o tamanho da escola, o números de alunos em sala de aula e o clima organizacional (Sakharov \& Farber, 1983).

No Ensino Especial, métodos, técnicas, currículo, recursos educativos e organização diferenciada devem ser aplicados em sala de aula para atender às necessidades específicas destes alunos (MEC, 2001). Cabe ressaltar que o trabalho no Ensino Especial tem algumas peculiaridades, 
como aprendizado heterogêneo dos alunos e necessidade de atender demandas diferenciadas em sala de aula, que são fatores de estresse (Antoniou, Polychroni, \& Walters, 2000), além de aspectos que demandam alta carga de emocional para o desenvolvimento do trabalho (Gil-Monte, Carretero, Roldán, \& Román, 2005). Este lugar ocupado pelo professor no Ensino Especial, em que muitas vezes ele exerce também a função de cuidador, exige responsabilidade dobrada e atenção constante, o que o, leva a envolver-se de forma intensa com seus alunos (Naujorks \& Barasuol, 2004).

Cargas elevadas de trabalho, processos burocráticos, reuniões constantes e desempenhos heterogêneos dos alunos podem ser citados como fontes de estresse nesta modalidade de ensino (Fore, Martin, \& Bender, 2002). É típico da função do docente de Ensino Especial lidar diariamente com alunos difíceis, obtendo menores sucessos, que, ademais, quando ocorrem, usualmente são pouco percebidos e reconhecidos. Os professores são confrontados cotidianamente com uma prática que não se associa aos resultados escolares concretos obtidos, o que os leva a desenvolver um conjunto cognitivo negativo e a percepção de que são inaptos para lidar com a demanda de aprendizagem. Em face disto, alguns optam por aposentar-se e outros diminuem a interação e o investimento no trabalho (Lavian, 2012; Qutaiba, 2011).

Qutaiba (2011) refere que professores do Ensino Especial apresentam crescente nível de insatisfação com o seu trabalho e o desejo de mudar de carreira. Estas manifestações têm chamado a atenção de psicólogos, que procuram fazer uma análise mais aprofundada desta situação, porquanto a insatisfação no trabalho demonstra ser um importante preditor da SB. Um estudo realizado por Carlotto, Librelotto, Pizzinato e Barcinski (2012) que investigou os fatores associados ao Burnout em professores do Ensino Especial identificou que o Perfil 1 da síndrome associava-se ao aumento do conflito de papel e ao sentimento de iniquidade, e o Perfil 2, ao conflito de papel, à sobrecarga de trabalho, à iniquidade, à insatisfação laboral e a problemas de saúde.

Pesquisas desenvolvidas com o objetivo de comparar Burnout em professores do Ensino Especial e do ensino regular têm identificado maiores níveis de Burnout no Ensino Especial (Beck \& Gargiulo, 1983; Kokkinos \& Panayiotou, 2005; Lavian, 2012; Qutaiba, 2011; Roach, 2009). Em consonância com o exposto, o presente estudo teve como objetivo verificar se existem diferenças entres os índices das dimensões da SB e os dos perfis 1 e 2 em professores do Ensino Especial e do ensino regular. O estudo em foco trabalha com a hipótese de que professores do Ensino Especial apresentam maiores índices nas dimensões e perfis da SB.

\section{Método}

\section{Participantes}

Participaram deste estudo 160 professores, dos quais 80 eram alocados no Ensino Especial (G1) de escolas da região do Vale do Rio dos Sinos e 80 professores, do Ensino Infantil e Fundamental (G2) de escolas regulares de Porto Alegre e região metropolitana. Os participantes do G1 foram recrutados por meio de um estudo realizado em 2011, e os do G2, em outro estudo, realizado em 2009. Os grupos foram pareados e comparados para atender ao objetivo do presente estudo.

Os professores do Ensino Especial (G1) são, em sua maioria, do sexo feminino (90\%), com companheiro fixo $(83,8 \%)$ e filhos $(60 \%)$. A idade média dos profissionais é de 36 anos $(D P=10,42)$. Quanto à formação, 80\% possuem formação em nível superior e $20 \%$ em nível de magistério. Contam, em média, doze anos de docência $(D P=9,22)$ e oito anos de atuação docente no Ensino Especial (DP=7,74), e atendem diariamente, em média, dezessete alunos $(\mathrm{DP}=13,07)$. A maioria trabalha exclusivamente na atual instituição (72,5\%). O grupo do ensino regular (G2) é, em sua maioria, constituído por mulheres (90\%), possui companheiro fixo $(71,8 \%)$ e filhos $(90 \%)$. Os profissionais têm idade média de 36 anos (DP=10,29). Destes, 84,7\% possuem formação em nível superior e 16,3\% em nível de magistério, e $71,3 \%$ não exercem atividade docente em outra instituição; contam doze anos de tempo de docência $(D P=9,31)$ e atuam no seu local de trabalho há sete anos $(\mathrm{DP}=7,20)$, atendendo diariamente, em média, 68 alunos ( $D P=91,34)$.

\section{Instrumentos}

Nesta pesquisa foram utilizados dois instrumentos, a saber: 1 - Questionário estruturado para coleta de dados demográficos (sexo, situação conjugal, formação, filhos, idade) e laborais (tempo de docência, número de alunos atendidos por dia, tempo de atuação na atual escola e eventual vínculo com outra instituição de ensino); 2 - "Cuestionario para la Evaluación del Síndrome de Quermarse por el Trabajo" para a avaliação da SB (CESQT), versão adaptada para o uso no Brasil por Gil-Monte, Carlotto e Câmara (2010). Este é formado por quatro dimensões, a saber: (I) llusão pelo trabalho; (II) Desgaste psíquico; (III) Indolência; e (IV) Culpa. O instrumento consta de vinte itens, que se distribuem em quatro subescalas, denominadas: Ilusão pelo Trabalho (cinco itens, alfa $=0,72$ ); Desgaste psíquico (quatro itens, alfa=0,86); Indolência (seis itens, alfa $=0,75$ ); e, Culpa (cinco itens, alfa = 0,79 ). Os itens são avaliados com uma escala tipo Likert de frequência de quatro pontos (0 "Nunca" a 4 "Todos os dias").

\section{Procedimentos}

A partir do banco de dados do $\mathrm{G} 1$, selecionou-se o mesmo número de participantes entre os 714 do banco de dados do G2, adotando-se como critério de pareamento as variáveis sexo, idade e tempo de docência. Do total de 88 professores do Ensino Especial, oito participantes foram excluídos por não apresentarem equivalência nos critérios utilizados. Sendo assim, para esta pesquisa constitui-se uma 
Tabela 1. Comparação de médias das dimensões da SB de professores de ensino regular e especial

\begin{tabular}{|c|c|c|c|c|c|}
\hline & Tipo de Ensino & $M$ & $D P$ & $P$ & $\begin{array}{l}\text { Effect size } \\
\text { Cohen's } d\end{array}$ \\
\hline \multirow{2}{*}{ Ilusão pelo trabalho } & Especial & 3,52 & 0,53 & $0,001^{*}$ & 0,8 \\
\hline & Regular & 2,96 & 0,80 & & \\
\hline \multirow{2}{*}{ Desgaste } & Especial & 1,47 & 0,99 & 0,457 & 0,1 \\
\hline & Regular & 1,59 & 0,99 & & \\
\hline \multirow{2}{*}{ Indolência } & Especial & 0,80 & 0,48 & 0,112 & 0,2 \\
\hline & Regular & 0,97 & 0,80 & & \\
\hline \multirow{2}{*}{ Culpa } & Especial & 1,13 & 0,66 & 0,689 & 0,0 \\
\hline & Regular & 1,09 & 0,76 & & \\
\hline \multirow{2}{*}{ SQT perfil1 } & Especial & 0,92 & 0,48 & $0,003^{*}$ & 0,5 \\
\hline & Regular & 1,20 & 0,68 & & \\
\hline \multirow{2}{*}{ SQT perfil2 } & Especial & 0,97 & 0,44 & $0,025^{*}$ & 0,4 \\
\hline & Regular & 1,17 & 0,66 & & \\
\hline
\end{tabular}

amostra intencional composta por 80 professores do Ensino Especial e 80 professores de Ensino Regular. É importante registrar que no espaço de tempo entre a coleta de dados do banco do G1 e do banco do G2 não há registros de alteração de grande impacto na orientação político-pedagógica das instituições avaliadas.

Ambos os estudos receberam aprovação de Comitês de Ética em Pesquisa, a saber: o estudo com professores do Ensino Especial foi aprovado pelo Comitê de Ética em Pesquisa da Pontifícia Universidade Católica do Rio Grande do Sul, n¹821/11, e o estudo dos docentes do Ensino Regular foram aprovados pelo Comitê de Ética em Pesquisa da Universidade Luterana do Brasil, n. ${ }^{\circ} 491 \mathrm{H}$. Todos os professores participantes desta pesquisa foram convidados a preencher o Termo de Consentimento Livre e Esclarecido e a pesquisa atendeu aos procedimentos éticos estabelecido pelo Conselho Nacional de Saúde (CNS, 1997).

A análise de dados foi realizada por meio do Statistical Package for the Social Sciences - SPSS versão 17.0. Inicialmente, foram executadas análises estatísticas de caráter descritivo e exploratório para avaliar a qualidade do banco de dados digitado e possíveis erros de digitação e de distribuição de itens, e para controlar as medidas utilizadas para o pareamento. O Perfil 1 constituiu-se da média dos quinze itens das subescalas de llusão pelo trabalho (subescala invertida), Desgaste psíquico e Indolência; e o Perfil 2 foi elaborado acrescentando-se a média da dimensão Culpa. Para a comparação de médias, foi utilizado o teste $t$ de student. Os resultados foram considerados estatisticamente significativos quando o valor de $p \leq 0,05$. O tamanho do efeito foi calculado pela diferença média padronizada entre dois grupos ( $d$ de Cohen) que considera 0,2 um valor indicativo de um efeito pequeno, 0,5 um efeito médio e 0,8 um tamanho de efeito grande (Cohen, 1992).

\section{Resultados}

Relativamente aos índices da SB, verificou-se que professores do Ensino Especial apresentam elevação nos índices da dimensão de llusão pelo trabalho. Ainda, em professores do Ensino Regular, há maiores índices nos perfis 1 e 2, conforme mostra a tabela 1. O tamanho do efeito calculado pelo $d$ de Cohen para os resultados significativos variou de 0,4 (médio efeito) a 0,8 (grande efeito).

\section{Discussão}

O objetivo deste estudo foi verificar se existem diferenças nos índices das dimensões de SB e perfis 1 e 2 no Ensino Regular e Especial, com a hipótese de que professores do Ensino Especial possuem índices mais elevados de Burnout. Os resultados não confirmam a hipótese da pesquisa, uma vez que os professores do Ensino Especial apresentam índices mais elevados da dimensão de llusão pelo trabalho e menores nos perfis 1 e 2 de Burnout. Este resultado está em desacordo com a maior parte dos estudos internacionais (Kokkinos \& Panayiotou, 2005; Lavian, 2012; Qutaiba, 2011; Roach, 2009), os quais indicam serem os professores do Ensino especial os que apresentam maiores níveis de Burnout, considerando-se a avaliação dos perfis, e 
corrobora o resultado do estudo nacional de Silva e Almeida (2011), o qual identificou diferença significativa apenas na dimensão Realização profissional, equivalente, neste estudo, à dimensão llusão pelo trabalho.

Pode-se entender este resultado a partir de questões culturais, pois crescem na literatura pesquisas atinentes à concepção de que estresse e Burnout podem ter diferentes configurações, a depender do contexto cultural, social e político da população em estudo (Etzion \& Pines, 1986; Reichel \& Neumann, 1993). Certos aspectos da manifestação de Burnout estão presentes em qualquer contexto sociocultural, embora, juntamente com as semelhanças, por vezes as manifestações assumam contornos específicos, determinados pelas particularidades do ambiente de trabalho, da organização e dos aspectos socioculturais mais amplos presentes nas diferentes sociedades (Moura, 1997; Vanheule, Rosseel, \& Vlerick, 2007).

Friedman (1991), ao estudar a relação entre aspectos culturais e Burnout em escolas israelenses, verificou que, nas instituições onde as políticas e normas eram mais claras, as vias de comunicação mais abertas e a estrutura hierárquica mais bem definida, era propiciado um clima organizacional favorável, em virtude do qual o trabalho desenvolvia-se de forma mais satisfatória. Essas questões foram consideradas pelo autor como variáveis significativas associadas a baixos níveis de Burnout. As instituições de Ensino Especial apresentam algumas especificidades quando comparadas com as instituições de Ensino Regular. De acordo com o estatuto das escolas de Ensino Especial do Rio Grande do Sul (APAE, 2006), este tipo de escola caracteriza-se por ser uma associação civil, filantrópica, de caráter educacional, cultural, assistencial, de saúde, de estudo e pesquisa, desportivo e outros, sem fins lucrativos. Possui um regimento próprio, no qual a direção é um membro eleito pela comunidade e parte de seus professores, técnicos e serviços de apoio são cedidos pela Secretaria de Educação do município, que escolhem deslocar-se para a instituição; outra parte é contratada pela Direção e pelo Conselho Administrativo e passa por um processo seletivo, no qual são verificadas questões relacionadas à motivação para o trabalho e à formação específica para o desenvolvimento de atividades docentes com a clientela.

Tal configuração de trabalho poderia ser uma das explicações para a maior llusão pelo trabalho nos professores do Ensino Especial. Kucuksileymanoglu (2011) e Acker (1999) afirmam que profissionais que possuem formação continuada adequada e expectativas realísticas em relação às suas atividades laborais têm menor probabilidade de desenvolver a SB. Professores que trabalham com alunos especiais, deficientes mentais e alunos com problemas sérios de desenvolvimento apresentam menores níveis de Burnout que os professores em geral, e têm sentimentos de competência interpessoal mais elevados e menores escores de despersonalização (Beck \& Gargiulo, 1983).

A formação implica, também, em maior sentimento de autoeficácia, a qual exerce influência nas ações realizadas, sendo um importante fator no processo de motivação. Uma vez que uma ação tenha sido tomada, as pessoas que se avaliam como competentes investem mais esforços e persistem por mais tempo em suas metas; além disso, escolhem realizar tarefas mais desafiadoras e tendem a fixar metas mais elevadas e antecipar cenários otimistas (Bandura, 1997). Assim, a crença otimista na própria competência para lidar com desafios diários aumenta a motivação para atuar de forma construtiva ante as demandas da profissão. No tocante a professores, esta questão é fundamental, pois as situações cotidianas, se avaliadas de forma menos ameaçadora, contribuem para um melhor desempenho profissional. A adaptação bem-sucedida às demandas estressantes impede o surgimento do Burnout (Brouwers, Tomic, \& Boluijt, 2011).

A formação continuada capacita o docente para a utilização de técnicas centradas na deficiência dos alunos e de recursos diretamente vinculados à tipologia e especificidades de cada aluno atendido (Michels, 2011), fazendo-o exercitar a criatividade e autonomia, aspectos relacionados à llusão pelo trabalho. No Ensino Regular ocorre uma formação mais generalista, tendo como ponto central a defasagem existente entre a formação oferecida pelas escolas e a realidade da atividade prática. Os processos de formação de educadores atendem a um modelo tecnicista ao tomarem como elemento principal a organização racional dos meios, as tecnologias e os procedimentos de ensino (Altenfelder, 2005). A falta de uma política escolar de formação continuada não possibilita que o professor articule suas atribuições com práticas criativas em relação ao aluno, à escola e à sociedade (Lima, 2001). Além disso, professores do Ensino Regular consideram inadequada a formação recebida para lidar com as atividades de ensino, escola e cultura institucional (Farber, 1991; Gatti, 2009; Lelis, 2001; Wisniewski \& Gargiulo, 1997). Segundo Farber (1991), a formação docente enfatiza conteúdos e tecnologias, sendo deficiente a abordagem das questões de relacionamento interpessoal, relacionamento com alunos, administradores e pais, e de outras situações que os professores sentem serem tão importantes quanto as outras.

O professor do Ensino Especial pode estar mais identificado com seu papel de educador. Edelwich e Brodsky (1980) referem que, de forma geral, uma das principais motivações das pessoas que trabalham em profissões de ajuda é o desejo pessoal de cuidar dos demais. Professores do Ensino Especial têm senso de idealismo e creem na sua capacidade de trabalhar arduamente para atingir seus objetivos (Lavian, 2012).

Outra questão que pode ser considerada específica da realidade do Ensino Especial é o menor número de alunos atendidos, variável frequentemente relacionada ao Burnout (Cordes \& Dougherty, 1993; Williams \& Gersch, 2004). Nas amostras do presente estudo, professores do Ensino Regular atendem, em média, 68 alunos diariamente, e os do Ensino Especial, dezessete alunos, o que indica uma maior possibilidade de acompanhamento e retorno. Estudo realizado por Goetzinger (2006) com professores do Ensino Especial estadunidenses constatou que os docentes que possuíam menor número de alunos apresentavam menores níveis de Burnout. 
Quanto aos perfis de Burnout, a pesquisa evidenciou menores índices dos perfis 1 e 2 em professores do Ensino Especial, fato que reforça a importância da dimensão da llusão pelo trabalho como um possível fator de proteção, uma vez que as demais dimensões não apresentaram diferenças significativas entre os grupos. O professor que declara ter escolhido a profissão docente devido à vocação é menos suscetível a apresentar baixa realização profissional e mais propenso a desenvolver maior exaustão emocional (Vercambre, Brossellin, Gilbert, Nerrière, \& Kovess-Masféty, 2009). Resultado semelhante foi encontrado em professores chilenos, pois os participantes que demonstravam uma visão mais positiva acerca do trabalho realizado, ou seja, concebiam-no como uma carreira e vocação, assinalaram menores níveis de Burnout (Ramírez-Pérez \& Zurita, 2010)

\section{Conclusão}

As diferenças identificadas entre professores do Ensino Especial e do Ensino Regular reforçam os resultados já encontrados por Pocinho e Capelo (2009) e Guglielmi e Tatrow (1998), os quais explicam que estas diferenças podem estar relacionadas à natureza do trabalho desenvolvido e ao tipo de aluno atendido, como também à cultura e ao contexto social, fatores determinantes para o desenvolvimento da SB. O estudo em foco destaca a importância do desejo individual para atingir metas relacionadas ao trabalho percebidas pelo sujeito como atraentes e fonte de satisfação pessoal, conforme o modelo de Burnout utilizado (Gil-Monte, 2005). O resultado obtido aponta a necessidade de aprofundamento em estudos que investiguem a relação entre Burnout e variáveis relacionadas à motivação e à identificação profissional. Também sugere a realização de estudos com variáveis que possibilitem a avaliação de preditores da SB para cada tipo de ensino.

Embora apresente pontos fortes - como a utilização de delineamento de grupos pareados -, e utilize instrumento adaptado com adequadas propriedades psicométricas, este estudo tem algumas limitações que devem ser consideradas na análise de seus resultados e conclusões. A primeira é que o estudo tem delineamento transversal, o que impede conclusões em termos de causalidade. A segunda diz respeito ao tipo de amostra, uma vez que a amostra investigada é não probabilística, portanto não é passível de generalizações para outras instituições ou profissionais. A terceira refere-se ao efeito do trabalhador sadio, questão peculiar em estudos transversais quanto à epidemiologia ocupacional, que, muitas vezes, exclui o possível doente (McMichael, 1976). Essa é uma situação que pode subestimar o tamanho dos riscos identificados, porque os mais afetados não conseguem manter-se no emprego ou se afastam para tratamento da saúde. A quarta é que foram utilizadas apenas medidas de autorrelato, o que pode ocasionar algum viés devido à desejabilidade social, que em algumas questões abordadas neste estudo está relacionada à dimensão de Indolência, pois é difícil para o profissional admitir que se distancie de seus alunos e os trate de forma impessoal.
Em termos de intervenção, pode-se pensar em ações diferenciadas entre os grupos: no Ensino Especial, em ações que reforcem o envolvimento com o trabalho; e, no regular, em ações que promovam uma implicação ativa na busca de uma formação continuada que possibilite reformulações na execução do trabalho, em busca de novas formas de realização profissional (Abalo \& Roger, 1998). Neste sentido, é importante dar atenção a políticas que estimulem e valorizem a profissão docente, permitindo que os professores manifestem sua competência e motivação profissional.

\section{Referências}

Abalo, J. G., \& Roger, M., C. (1998). Calidad de vida y salud. Problemas actuales en su investigación. Burnout: una amenaza a los equipo de la salud. En Alapsa-Asociación Latinoamericana de Psicología de la Salud, 2. Recuperado: 01 ago 2012. Disponível: http:// www.alapsa.org.com

Acker, G. M. (1999). The impact of client's mental illness on social worker's job satisfaction and burnout. Health and Social Work, 24(2), 112-120.

Altenfelder, A. H. (2005). Desafios e tendências em formação continuada. Construção Psicopedagógica, 13(10). Recuperado: 01 ago 2012. Disponível: <http://pepsic.bvsalud.org/scielo. php?script=sci_arttext\&pid=S1415 69542005000100004\&lng=pt\& nrm=iso>. ISSN 1415-6954.

Antoniou, A. S., Polychroni, F., \& Walters, B. (2000). Sources of stress and Professional burnout of teachers of special educational needs in Greece. International Special Education Congress, University of Manchester.

APAE - Associação de Pais e Amigos dos excepcionais (2006). Estatuto da Federação das Apaes do Rio Grande do Sul. Recuperado: 01 ago 2012. Disponível: http://www.apaebrasil.org. br/federacoes-estaduais.phtml

Bandura, A. (2007). Self-efficacy: the exercise of control. New York: W. H. Freeman and Company.

Batista, J. B. V., Carlotto, M. S., Coutinho, A. S., \& Augusto, L. G. S. (2010). Prevalência da Síndrome de Burnout e fatores sociodemográficos e laborais em professores de escolas municipais da cidade de João Pessoa, PB. Revista Brasileira de Epidemiologia, 13(3), 502-512.

Beck, C. L., \& Gargiulo, R. M. (1983). Burnout in teachers of retarded and non-retarded children. Journal of Educational Research, 76, 169-173.

Resolução CNE/CEB n², de 11 de fevereiro de 2001. (2001). Estabelece as Diretrizes Curriculares Nacionais para o Ensino Especial. Brasília: MEC/SEF. 
Brouwers, A., Tomic, W., \& Boluijt, H. (2011). Job demands, job control, social support and self-efficacy beliefs as determinants of burnout among physical education teachers. Europe's Journal of Psychology, 7(1), 17-39.

Carlotto, M. S. (2010). Síndrome de Burnout: O estresse ocupacional do professor. Canoas, RS: Editora Ulbra.

Carlotto, M. S., Librelotto, R., Pizzinato, A., \& Barcinski, M. (2012). Prevalência e fatores associados à Síndrome de Burnout em professores de ensino especial. Análise Psicológica, 30, 315-327.

Cherniss C. (1980). Professional burnout in human service organizations. New York: Praeger.

Cordes, C. L., \& Dougherty, T. W. (1993). A review and an integration of research on job burnout. Academy of Management Review, 18(4), 621-656.

Crane, S. J., \& Iwanicki, E. F. (1986). Perceived role conflict, role ambiguity, and burnout among special education teachers. Remedial and Special Education, 7(2), 24-31.

Edelwich, J., \& Brodsky, A. (1980). Burnout: stages of disillusionment in the helping profession. New York: Human Sciences Press.

Etzion, D., \& Pines, A. (1986). Sex and culture in burnout and coping among human service professionals: a social psychological perspective. Journal of Cross-Cultural Psychology, 17(2), 191-209.

Farber, B. A. (1991). Crisis in education. Stress and burnout in the American teacher. San Francisco: Jossey-Bass Inc.

Friedman, I. A. (1991). High and low burnout schools: school culture aspects of teacher burnout. Journal of Educational Research, 84(6), 325-333.

Fore, C., Martin,C., \& Bender, W. N. (2002). Teacher burnout in special education: the causes and the recommended solutions. The High School Journal, 86(1), 36-44.

Gasparini, S. M., Barreto, S. M., \& Assunção, A. A. (2006). Prevalência de transtornos mentais comuns em professores da rede municipal de Belo Horizonte, Minas Gerais, Brasil. Cadernos de Saúde Pública, 22(12), 2679-2691.

Gatti, B. A. (2009). Formação de professores: condições e problemas atuais. Revista Brasileira de Formação de Professores, 1(1), 90-102.

Gil-Monte, P. R. (2005). El síndrome de quemarse por el trabajo. Madrid: Pirâmide.

Gil-Monte, P., Carretero, N., Roldán, M.. D., \& Nuñes-Roman, E. V. (2005). Prevalência del síndrome de quemarse por el trabajo (burnout) em monitores de taller para personas com descapacidad. Revista de Psicologia del Trabajo y de las Organizaciones, 21(12), 107-123.
Gil-Monte, P. G., Carlotto, M. S., \& Câmara, S. G. (2010). Validação da versão Brasileira do "Cuestinario para La Evaluación del Síndrome de Quemarse por El Trabajo" em professores. Revista de Saúde Pública, 44(1), 140-147.

Goetzinger, E. K. (2006). Burnout among special educators: Do experience, certification, caseload, and school size make a difference? Oklahoma: The University of Oklahoma.

Gomes, A. R., Montenegro, N., Peixoto, A. M. B. C., \& Peixoto, A. R. B. C. (2010). Stress ocupacional no ensino: um estudo com professores do $3^{0}$ ciclo e ensino secundário. Psicologia \& Sociedade, 22(3), 67-93.

Guglielmi, R. S., \& Tatrow, K. (1998). Occupational stress, burnout, and health in teachers: a methodological and theoretical analysis. Review of Educational Research, 68(1), 61-69.

Kokkinos, C., \& Panayiotou, G. (2005). Correlates of teacher appraisals of student behaviours. Psychology in the School, 42, 79-89.

Kucuksileymanoglu, R. (2011). Burnout syndrome in levels of teachers in special education school in Turkey. International Journal of Special Education, 26(1). Recuperado: 01 ago 2012. Disponível: http://www.eric.ed.gov/PDFS/EJ921186.pdf

Lavian, R. H. (2012). The impact of organizational climate on burnout among homeroom teachers and special education teachers (full classes/individual pupils) in mainstream schools. Teachers and Teaching: Theory and Practice, 18(2). 233-247.

Lelis, I. A. (2001). Do ensino de conteúdos aos saberes do professor: mudança de idioma pedagógico?. Revista Educação e Sociedade, $74,43-58$.

Leite, M. de P., \& Souza, A. N. de (2007). Condições do trabalho e suas repercussões na saúde dos professores da educação básica no Brasil - Estado da Arte. São Paulo: Fundacento/Unicamp.

Lima, M. S. L. (2001). A formação contínua do professor nos caminhos e descaminhos do desenvolvimento profissional. Tese de doutorado, Faculdade de Educação, Universidade de São Paulo, São Paulo.

Maslach, C. (2003). Burnout: the cost of caring. Cambridge: Malor Books.

Maslach, C., Schaufeli, W. B., \& Leither, M. P. (2001). Job burnout. Annual Rewiew Psychology, 52, 397-422.

McMichael, A. J. (1976). Standardized mortality ratios and the healthy worker effect: scratching beneath the surface. Journal Occupational Medical, 18, 165-168.

Michels, M. H. (2011). O que há de novo na formação de professores para a educação de Ensino Especial? Revista de Educação Especial, 24(40), 219-232. 
Resolução CNS 196/196. (1997). Conselho Nacional de Saúde. Diretrizes e normas para pesquisa envolvendo seres humanos. Brasília: Ministério da Saúde.

Moura, E. P. G. (1997). Saúde mental e trabalho. Esgotamento profissional em professores da Rede de Ensino Particular de Pelotas - RS. Dissertação de Mestrado, Pontifícia Universidade Católica do Rio Grande do Sul, Porto Alegre, Rio Grande do Sul.

Naujorks, M. I., \& Barasuol, E. B. (2004). Burnout docente no trabalho com a inclusão de alunos com necessidades educativas especiais. Cadernos de Educação Especial, 24, 97-106.

Organização Mundial da Saúde (1994). Global Strategy on Occupational Health for All: The way to health at work. Organización Mundial de Salud. Recuperado: 01 ago 2012. Disponível: http:// www.medicinanews.trra.co.cr

Pocinho, M., \& Capelo, M. R. (2009). Vulnerabilidade ao estresse, estratégias de coping e autoeficácia em professores portugueses. Revista Educação e Pesquisa, 35(2), 351-367.

Pocinho, M., \& Parestrelo, C. X. (2011). Um ensaio sobre burnout, engagement e estratégias de coping na profissão docente. Revista Educação e Pesquisa, 37(3), 513-528.

Qutaiba, A. (2011). The relationship between the Level of SchoolInvolvement and Learned Helplessness among Special- Education Teachers in the Arab Sector. Australian Journal of Teacher Education, 36(2), 1-15.

Ramírez-Pérez, M., \& Zurita, R. Z. (2010). Variables organizacionales y psicosociales asociadas al síndrome de burnout en trabajadores del ámbito educacional. Revista de la Universidad Bolivariana, 9(25), 515-534.

Roach, A. (2009). Teacher Burnout: Special Education versus Regular Education. Education Specialist in School Psychology, Marshall University, USA.

Reichel, A., \& Neumann, Y. (1993). Work stress, job burnout, and work outcomes in a turbulent environment. International Studies of Management \& Organization, 23(3), 75-97.
Rudow, B. (1999). Stress and burnout in the teaching profession: european studies, issues, and research perspectives. Em R. Vanderbergue \& M. A. Huberman (Eds.), Understanding and preventing teacher burnout: a source book of international practice and research (pp.38-58). Cambridge: Cambridge University Press.

Sakharov, M., \& Farber, B. (1983). A critical study of burnout in teachers. Em B. A. Farber (Eds.), Stress and burnout in the human service professions (pp. 65-81). New York: Pergamon.

Silva, N. R., \& Almeida, M. A. (2011). As características dos alunos são determinantes para o adoecimento de professores - Um estudo comparativo sobre a incidência de Burnout em professores do ensino regular e especial. Revista Brasileira de Educação Especial, 17(3), 373- 394.

Vanheule, S., Rosseel. Y., \& Vlerick P. (2007). The factorial validity and measurement invariance of the Maslach Burnout Inventory for human services. Stress and Health, 23, 87-91.

Vercambre, M. N., Brosselin, P., Gilbert, F., Nerrière, E., \& KovessMasféty, V. (2009). Individual and contextual covariates of burnout: a cross-sectional nationwide study of French teachers. BMC Public Health, 9, 333. Recuperado: 30 set 2012. Disponível: http://www. biomedcentral.com/1471-2458/9/333

Williams, M., \& Gersch, I. (2004). Teaching in mainstream and special schools: are the stress similar or different? Britsh Journal of Special Education, 31(3), 157-162.

Wisniewski, L., \& Gargiulo, R. M. (1997). Occupational stress and burnout among special educators: a review of the literature. The Journal of Special Education, 31(3), 325-349.

Yong, Z., \& Yue, Y. (2007). Causes for burnout among secondary and elementary school teachers and preventive strategies. Chinese Education and Society, 40(5), 78-85.

Zabel, R. H., \& Zabel, M. K. (2001). Revisiting burnout among special education teachers: do age, experience, and preparation still matter? The Journal of the Teacher Education, 24(2), 128-139.

Recebido em: 03/08/2012

Reformulado em: 23/01/2013

Aprovado em: 28/01/2013 


\section{Sobre as autoras}

Ana Claudia Braun (anaclaudiabraun@hotmail.com)

Mestre em Psicologia Social pela Pontifícia Universidade Católica do Rio Grande do Sul

Mary Sandra Carlotto (mary.sandra@pucrs.br)

Professora do curso de Pós Graduação em Psiciologia Social da Pontifícia Universidade Católica do Rio Grande do Sul

Burnout em professores * Ana Claudia Braun \& Mary Sandra Carlotto 